Offizielles Organ folgender Verbände

Verband der Osteopathen Deutschland e.V. (VOD)

Deutsche Akademie für Osteopathische Medizin e.V. (DAOM)

D Association Luxembourgeoise des Ostéopathes (A.L.D.O.)

ISSN 1610-5044

4/2004, 2. Jahrgang

Herausgeber:

Prof. Dr. rer. nat. med. habil. Rainer Breul D.O.h.c.

Marina Fuhrmann D.O. M.R.O.

Prof. Dr. med. habil. Karl-Ludwig Resch

Dr. med. Roger Seider D.O.

Redaktioneller Beirat:

Jo Buekens, Osteopath D.O., College Sutherland, Garnich,

Luxemburg

Prof. Dr. med. Jochen Fanghänel, Institut für Anatomie,

Ernst-Moritz-Arndt-Universität, Greifswald

Dr. med. Edgar Hinkelthein, Osteopath D.O., M.R.O., B. Sc. (Hons.)

Osteopathieschule Damp, Fleckeby

Werner Langer, Osteopath D.O., IfAO - Institut fü

Angewandte Osteopathie, Bitburg

Dr. med. Horst P. Schwerdtner, DAOM, Hamm

Wissenschaftlicher Beirat:

Prof. Dr. med. Jan Cabri, Centro de Est. de Fisioterapie, Faculdade

de Motricidade Hum., Lissabon, Portugal

Prof. Anthony G. Chila D.O. FAAO, College of Osteopathic

Medicine, Ohio University, Athens, Ohio USA

Christian Fossum D.O., European School of Osteopathy (ESO),

Maidstone, England

Dr. Viola M. Frymann D.O. FAAO, FCA;

Director of the Osteopathic Center for Children, San Diego,

Kalifornien USA

Kalifornien USA

Universität Rostock

Universität Rostock
Renzo Molinari, Principal of the European School for Osteopathy

(ESO), Maidstone, England

Dr. med. Heike Philippi, Kinderneurologin, Schlangenbad

Prof. Frank H. Willard Ph. D., College of Osteopathic Medicine,

University of New England, Biddeford, ME USA

Verlag

Hippokrates Verlag in

MVS Medizinverlage Stuttgart GmbH \& Co.KG

Oswald-Hesse-Str. 50, 70469 Stuttgart

Redaktion (v.i.S.d.P.):

Marina Horbatsch

Fon/Fax: (07 11) 8931-715/705

E-Mail: marina.horbatsch@medizinverlage.de

Christoph Newiger in team 4 U Medienbüro

Fraunhoferstr. 42, 80469 München

Fon/Fax: (089) 209001-67/-68

E-Mail: redaktion.do@medizinverlage.de

Marketing

Marion Krubasik

Fon/Fax: (07 11) 89 31-735/748

E-Mail: marion.krubasik@medizinverlage.de

Anzeigen:

Martin Riedel, MVS Mediaservice

Fon/Fax: (07 11) 8931-734/-706

E-Mail: martin.riedel@medizinverlage.de

Abonnementservice:

Fon/Fax: (07 11) 8931-321/-422

E-Mail: aboservice@thieme.de

Probeabonnement:

Fon/Fax: (07 11) 8931-906/-901

E-Mail: kundenservice@thieme.de

Produktion:

Satz \& Repro: Fotosatz Sauter, Donzdorf

Druck \& Bindung: Rondo Druck, Ebersbach

Autorenhinweise:

Auf Anfrage bei der Redaktion.

Erscheinungsweise:

Vierteljährlich

Bezugspreise 2005:

$\begin{array}{llll} & \begin{array}{l}\text { Abonnement- } \\ \text { preis }\end{array} & \begin{array}{l}\text { Versand- } \\ \text { kosten }\end{array} & \text { Gesamt } \\ \text { Inland } & € 67,90 & € 7,20 & € 75,10 \\ \text { Europa } & € 67,90 & € 12,90 & € 80,80\end{array}$

Restl. Welt $€ 67,90 \quad € 21,50 \quad € 89,40$

Aus-, Fort- und Weiterbildung, Studenten:

$\begin{array}{llll}\text { Inland } & € 41,90 & € 7,20 & € 49,10 \\ \text { Europa } & € 41,90 & € 12,90 & € 54,80 \\ \text { Restl. Welt } & € 41,90 & € 21,50 & € 63,40\end{array}$

Fortsetzung auf $S .2$

\section{Danke, Sz!}

Sz war „DIE“ Seiz. Dorothee Seiz, Cheflektorin beim Hippokrates Verlag. Mit „Ihre Sz“ endeten stets die E-Mails an alle, die mit ihr zusammenarbeiteten. Sz war der kreative Motor des Verlags, „Frau Hippokrates“, verantwortlich für die Naturheilkunde - nicht nur, aber im Laufe der Jahre immer mehr.

Sz war die Entdeckerin der Talente „ihrer“ Autoren, die sie motivierte, herausforderte und sich in sie hineinlebte, um die besten Seiten aus ihnen herauszuholen. Sie konnte gut das Gefühl vermitteln, dass man der wichtigste Autor sei, den der Verlag hat - und das für den Verlag alles entscheidende Buch schreibt. Im Sinne des Lesers stellte sie sich völlig in den Dienst eines Autors und des jeweiligen Buches. Sie war die erste Leserin. Seite für Seite ging sie durch - forderte eine Sprache, die auch andere verstehen. Mit Kreativität, Behutsamkeit und wohltuendem, echtem Interesse ließ sie eine berufliche Nähe entstehen, die die Autoren durch die Arbeit trug.

Berühmt waren ihre Scribbles, Zeichenentwürfe und Ideen, die dem Grafiker als Vorlage dienten, Mitarbeiter ermunterten oder ermahnten, sowie manch brisante Situation entschärften. Sie liebte es beispielsweise, die Wichtigkeit von „Brandbriefen“ durch Strichzeichnungen zu unterstreichen: Sz als Lektorin mit Dutt und Brille, die in ihrer Not oder aus Verzweiflung in den Stuttgarter Neckar springt, bzw. in ein Fass, oder sich in einem Mauseloch verkriecht.

Mit Sz konnte man herrlich streiten, im Streit auf eine Blödelei, ein Wortspiel verfallen - um dann wieder von ihr auf den Punkt gebracht zu werden. Gespräche und Verhandlungen führte sie taktisch äußerst geschickt, diplomatisch und hartnäckig. Für das, was ihr wichtig war, konnte sie kämpfen wie ein Drache.

Ihr eigen war die fanatische Liebe zur Arbeit und zum Einsatz, die totale Identifikation. $\mathrm{Zu}$ Unzeiten war sie im Verlag erreichbar, scheute weder Mühen noch weite Reisen. Selbst nach einem Kongresstag forschte sie beim Abendessen hellwach mit geradezu investigativen Fragen nach Trends im jeweiligen Naturheilkunde-Segment. Für ihre Verdienste um die Naturheilverfahren zeichnete sie der „Zentralverband der Ärzte für Naturheilverfahren“ Anfang der 1990er Jahre mit der goldenen Ehrennadel aus.

Persönliches ist wenig bekannt. Selbst leisesten Versuchen, sie hierzu auszuhorchen, begegnete sie mit einem charmanten Lächeln, mit vielsagendem Schweigen oder sie brachte ihre Gesprächspartner mit bestimmendem Ton wieder auf das geschäftliche Thema. Deswegen soll hier die „private“ Dorothee Seiz auch nicht interessieren.

Die DO ist ein junges Kind der Sz. Zusammen mit Frau Horbatsch brachte sie das Projekt noch auf den Weg, freute sich „wie verrückt“, als das Herausgeber-/RedakteurTeam wie erhofft das erste Mal zusammen kam und nordete die gesamte Mannschaft ein. Die Zeitschrift trägt unverkennbar ihre Handschrift. Am 15. Juli 2004 verstarb Dorothee Seiz. Die DO sagt: Danke, Sz!

Die Herausgeber

und stellvertretend für die Autoren des Hippokrates Verlages

Volker Fintelmann, Markus Wiesenauer, Beate Strittmatter und Karlheinz Bayer. 